ARTICLE

DOI: $10.1038 /$ ncomms10914 OPEN

\title{
Metal-free intermolecular formal cycloadditions enable an orthogonal access to nitrogen heterocycles
}

Lan-Gui Xie ${ }^{1}$, Supaporn Niyomchon ${ }^{1}$, Antonio J. Mota ${ }^{2}$, Leticia González ${ }^{2}$ \& Nuno Maulide ${ }^{1}$

Nitrogen-containing heteroaromatic cores are ubiquitous building blocks in organic chemistry. Herein, we present a family of metal-free intermolecular formal cycloaddition reactions that enable highly selective and orthogonal access to isoquinolines and pyrimidines at will. Applications of the products are complemented by a density functional theory mechanistic analysis that pinpoints the crucial factors responsible for the selectivity observed, including stoichiometry and the nature of the heteroalkyne.

\footnotetext{
${ }^{1}$ Institute of Organic Chemistry, University of Vienna, Währinger Strasse 38, 1090 Vienna, Austria. ${ }^{2}$ Institute of Theoretical Chemistry, University of Vienna, Währinger Strasse 17, 1090 Vienna, Austria. Correspondence and requests for materials should be addressed to N.M. (email: nuno.maulide@univie.ac.at).
} 
eteroarenes constitute one of the privileged core structural motifs in organic chemistry ${ }^{1}$. Among them, isoquinolines and pyrimidines represent two big families in pharmaceutical agents, natural products and functional materials $^{2-9}$. Therefore, continued effort is devoted to the exploration of new and efficient synthetic strategies for these backbones.

The classical strategies to prepare isoquinolines (Fig. 1a) generally focus on the crucial textbook disconnections C1-C8a (Bischler-Napieralski and Pictet-Spengler syntheses) or C4-C4a (Pomeranz-Fritsch synthesis). Recently developed routes centred on the bond-forming events $\mathrm{N} 2-\mathrm{C} 3$ or N2-C3/C4-C4a, employing electrophile-triggered annulation and transition metalcatalysed $\mathrm{C}-\mathrm{H}$ or $\mathrm{C}-$ halogen bond activation, respectively ${ }^{1}{ }^{10}{ }^{10}$ 17. A strategy relying on the simultaneous formation of $\mathrm{N} 2-\mathrm{C} 3 /$ $\mathrm{C} 1-\mathrm{C} 8 \mathrm{a}$ is much less documented ${ }^{18}$.

Conversely, most of the known avenues towards pyrimidine synthesis rely on the condensation of $\mathrm{N}-\mathrm{C}-\mathrm{N}$ subunits (mostly amidines or guanidines) with 1,3-dicarbonyl derivatives or the stoichiometric activation of carbonyl moieties with triflic anhydride (Fig. 1a) (refs 19-23). Ynamides have recently shown to be suitable candidates for regioselective cycloaddition with nitriles in the presence of a gold catalyst, leading to 4-aminopyrimidine $\operatorname{cores}^{24}$. Although the reactivity of ynamides has received considerable recent attention ${ }^{25-33}$, analogous investigation of the potential enclosed in the triple bond of thioalkynes is surprisingly $\operatorname{rare}^{34-37}$, even though the resulting sulfide is a useful ${ }^{38}$ and versatile substituent ${ }^{39-42}$.

Herein we report a family of reactions that enable a high yielding, orthogonal access to either isoquinolines or pyrimidines at will (Fig. 1b), by Brønsted acid-mediated regioselective formal cycloaddition of ynamides and thioalkynes with nitriles (for a review of transition-metal mediated $[2+2+2]$ cycloadditions) (ref. 43). Mechanistic studies reveal the subtle differences that are responsible for selectivity.

\section{Results}

Synthesis. Initial experiments involving the reaction of ynamide 1a with various Brønsted acids in the presence of varying amounts of acetonitrile led to moderate yields of isoquinoline 3aa. After optimization of conditions (see Supplementary Table 1 for details), we found that essentially equimolar amounts of $\mathbf{1 a}, \mathbf{2 a}$ and $\mathrm{TfOH}$ in dichloroethane as solvent sufficed to enable preparation of 3aa in $89 \%$ yield (for a discussion of stoichiometry in these reactions, vide infra).

Holding suitable conditions in hand, we then examined several nitriles 2a-j under the optimized conditions. As shown in Fig. 2a, this direct formal cycloaddition is applicable to a broad range of substrates, generally affording good to excellent yields of isoquinoline products. Remarkably, alkyl nitriles bearing

State-of-the-art methods for construction of isoquinolines and pyrimidines

Classical disconnections

Recently developed disconnections

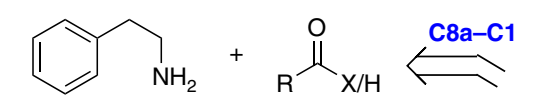

Bischler-Napieralski Pictet-Spengler
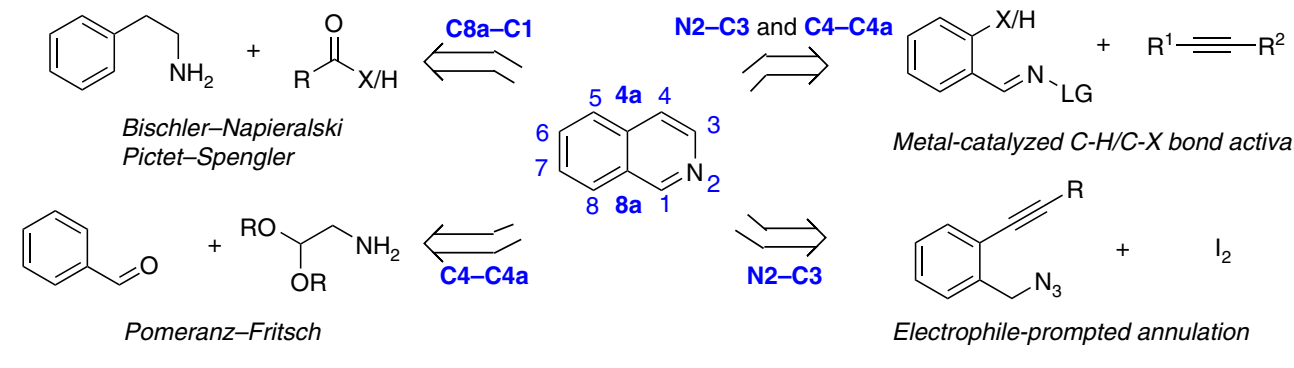

Metal-catalyzed C-H/C-X bond activation
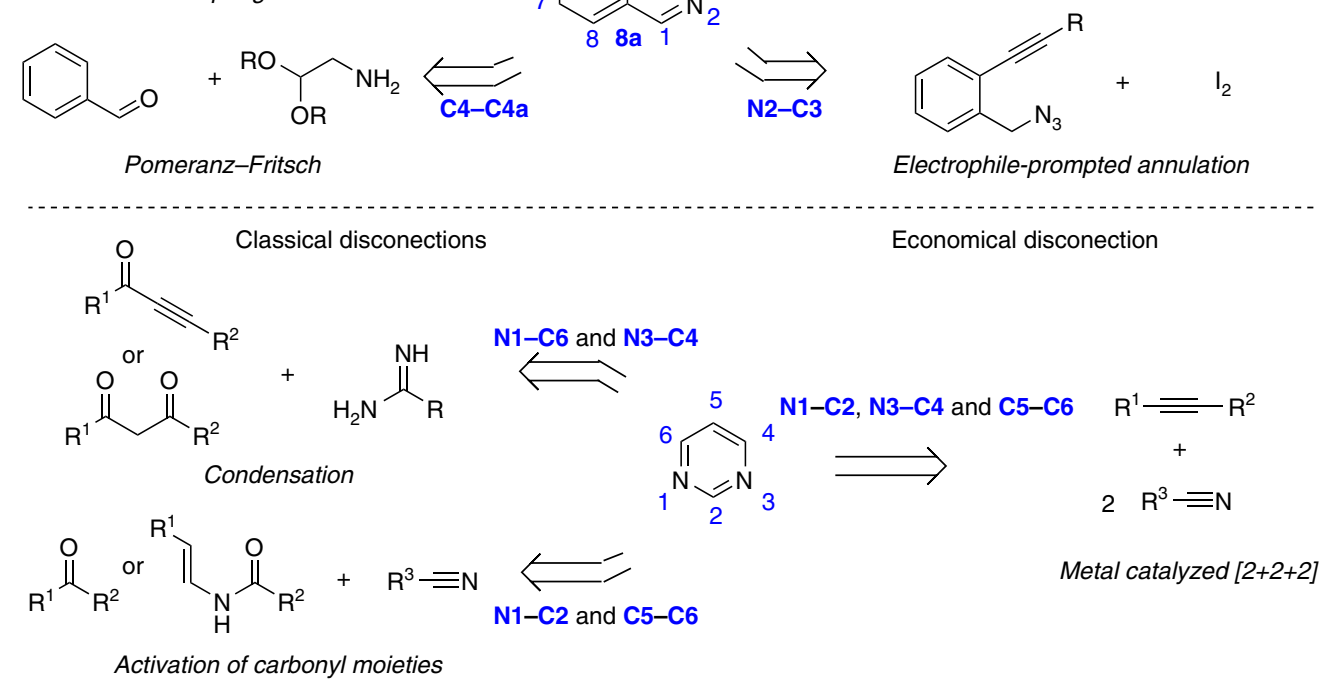

\section{b}

Our proposed disconnections (this work)

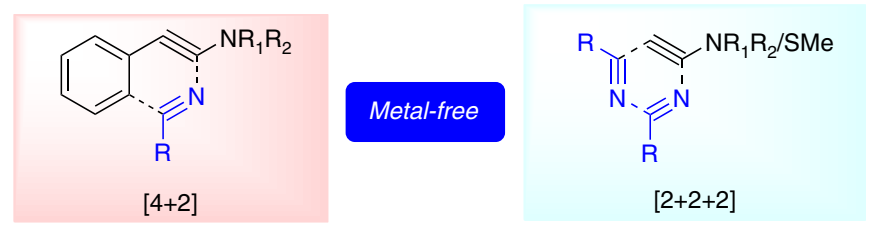

Figure 1 | Synthetic disconnections. (a) Known synthetic disconnections for the isoquinoline and pyrimidine backbone. (b) Proposed direct disconnections through intermolecular metal-free alkyne/nitrile cycloadditions. 
a

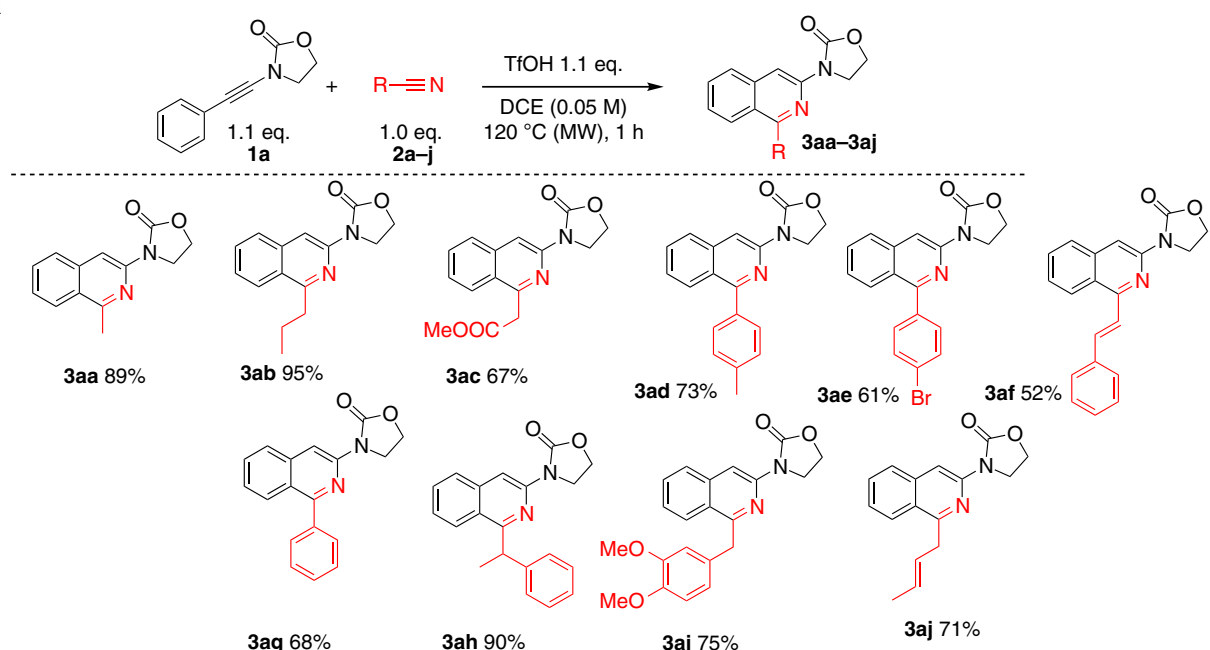

b

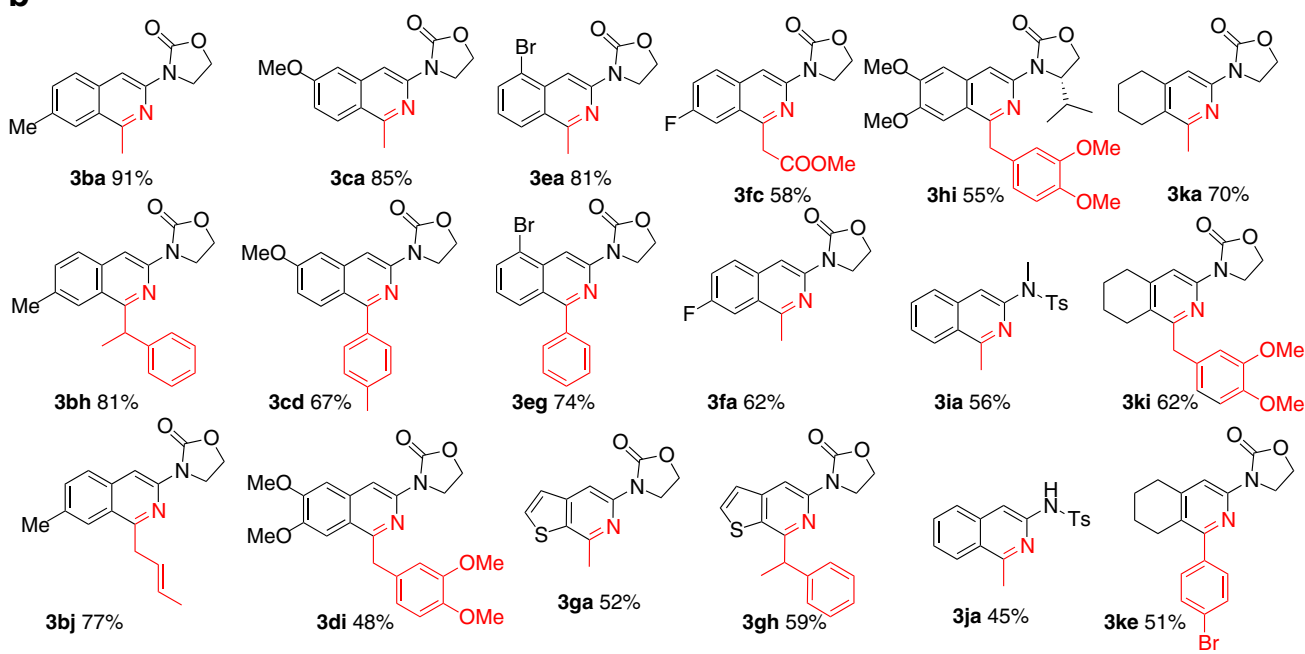

Figure 2 | Scope of isoquinoline synthesis. (a) Scope of nitriles and (b) Scope of ynamides for the synthesis of isoquinolines. Yields are for isolated products.

functional groups such as an ester (2c), aryl rings ( $2 \mathbf{h}$ and $2 \mathbf{i}$ ) or $\mathrm{C}-\mathrm{C}$ double bonds $(\mathbf{2} \mathbf{j})$ are compatible with the reaction conditions. It is worth mentioning that the isolated double bond in product 3aj does not migrate into conjugation with the isoquinoline ring under these conditions. Aryl nitriles $(\mathbf{2 d -}-\mathbf{g})$ and $\alpha, \beta$-unsaturated nitrile $\mathbf{2 f}$ are also viable partners delivering the corresponding substituted isoquinoline products in good to very good yields.

Subsequently, a broad range of ynamides were submitted to this protocol (Fig. 2b). In the event, both electron-donating (1b-d) and -withdrawing (1f) substituents were tolerated on the ynamide partner, leading to smooth isoquinoline assembly in good yields. Halogenated ynamides (1e-f) were also amenable to this reaction, delivering isoquinoline products ripe for subsequent divergent functionalization. Thienopyridine skeletons could be obtained in reasonable yields (3ga and 3gh). Interestingly, $N$-tosyl- $N$-benzyl ynamide (1j) directly generated the corresponding debenzylated product: the tosyl-protected, pharmacologically relevant 3-aminoisoquinoline (3ja) ( $r e f s ~ 44,45)$. Moreover, the use of an alkenyl-substituted ynamide (1k) led to the annulated pyridine product (3ke).

After this initial success, we hypothesized that other heteroatom-substituted alkynes might prove amenable to a similar modular assembly of isoquinolines. In particular, we were drawn to the use of thioalkynes such as $\mathbf{4 b}$, with the expectation of obtaining an (alkylthio)-isoquinoline 6ba where the sulfur residue could serve as a useful synthetic handle (Fig. 3a).

Much to our surprise, treatment of $\mathbf{4 b}$ with acetonitrile $\mathbf{2 a}$ under conditions identical to those employed previously led exclusively to the pyrimidine $\mathbf{5 b a}$ in $52 \%$ yield (Fig. 3a). Remarkably, product $\mathbf{5 b a}$ is the result of a formal, regioselective cycloaddition of one molecule of $\mathbf{4 b}$ with two molecules of $2 \mathbf{a}$. This dramatic shift in product selectivity between ynamides and thioalkynes eventually presented us with a versatile cycloaddition route towards pyrimidines. Reaction optimization showed that this transformation proceeds most effectively at room temperature in the presence of an excess of acetonitrile (see Supplementary Table 2 for details).

Figure $3 \mathrm{~b}$ depicts the full scope of nitriles $\mathbf{2 b}-\mathbf{v}$ compatible with this metal-free pyrimidine synthesis. Secondary aliphatic (2k) and alicyclic (2m-2p) carbonitriles smoothly coupled with thioalkyne 4a under the reaction conditions. This formal cycloaddition was also tolerant of nitriles bearing triple (2q) and double bonds $(\mathbf{2} \mathbf{j})$, including conjugated olefins (2f). Both electron-rich (2d and 2s) and electron-deficient $(\mathbf{2 t}$ and $\mathbf{2} \mathbf{u})$ substituted benzonitriles could be employed, providing the desired pyrimidine products in good to excellent yields. It is worth noting that heteroarylnitriles such as 3-cyanothiophene (2r) were also tolerated. The possibility 
a

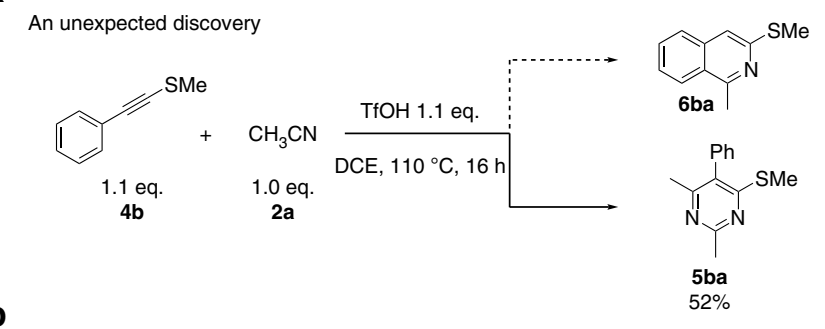

Scope of nitriles

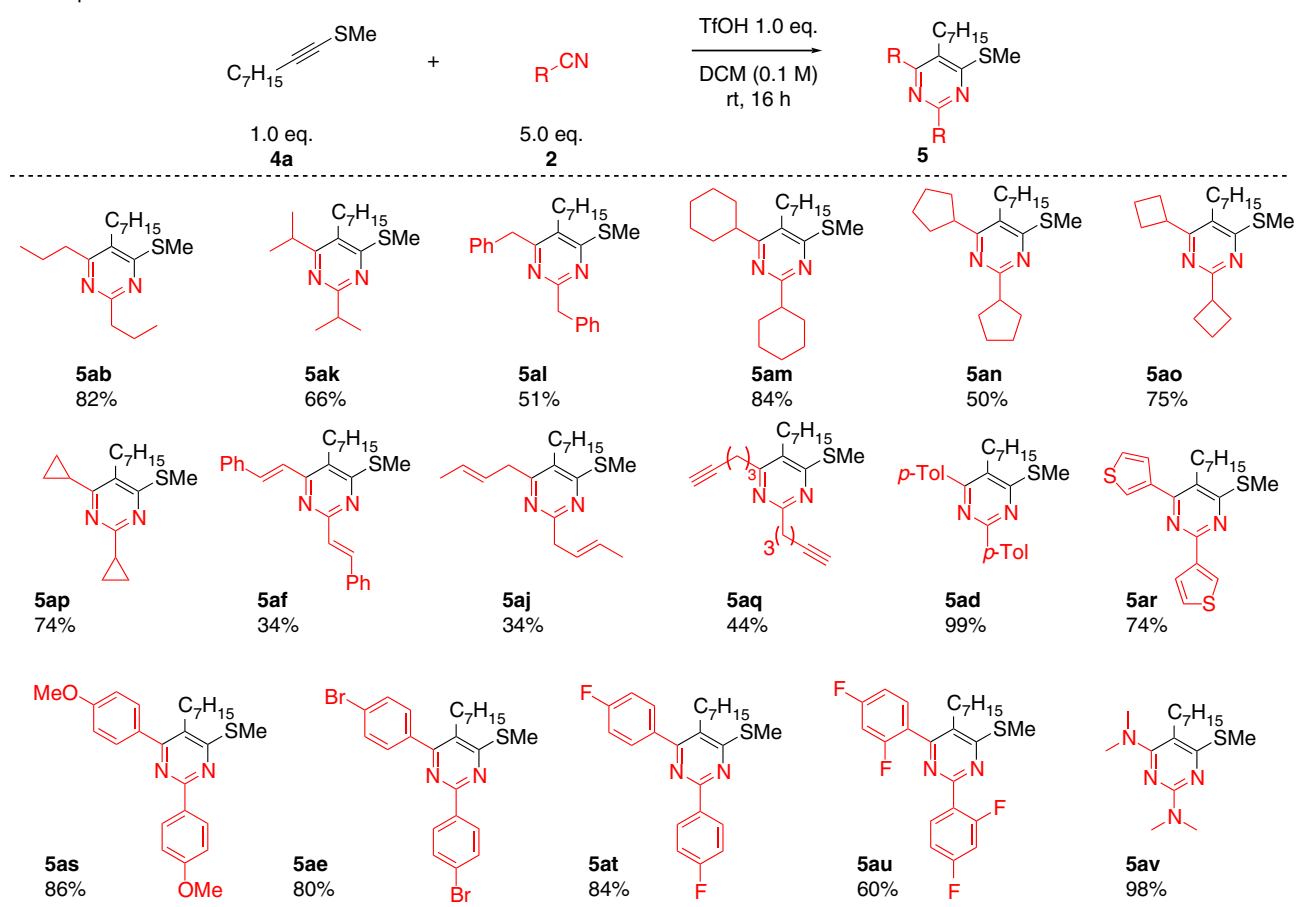

Figure 3 | Synthesis of pyrimidine. (a) Unexpected synthesis of pyrimidine $\mathbf{5 b a}$. (b) Scope of nitriles in the synthesis of pyrimidines $\mathbf{5}$.

of using dimethylcyanamide (2v), delivering an aminated pyrimidine in excellent yield, further highlights the generality of this synthetic method. Pyrimidine 5ae yielded crystals suitable for X-ray diffraction analysis, unambiguously confirming its structure (see Supplementary Fig. 64 and Supplementary Tables 4 and 5 for details).

Further studies focused on the scope of heteroalkynes for this pyrimidine synthesis (Fig. 4a). We were pleased to find that a cyclopropyl substituent (4c) was tolerated, as a cyclopropyl appended to a pyrimidine ring is a common feature in drug-like, biologically active cores ${ }^{46-48}$. Both electron-rich (4e and $\mathbf{4 g}$ ) and electron-poor (4d and $\mathbf{4 f}$ ) arylalkynes afforded the corresponding pyrimidine products in good yields. Furthermore, considerable flexibility can be exerted, concerning the location of substituents on the aryl ring $(\mathbf{4 d}-\mathbf{g})$.

Strikingly, we found that 4-aminosubstituted pyrimidines can also be obtained by exposing ynamides (11, 1a and $\mathbf{1 m}$ ) to the standard conditions developed for pyrimidine synthesis. A distal nitrile group carried by the ynamide partner could be successfully introduced into the pyrimidine product ( $7 \mathbf{m a})$. Remarkably, when phenyl-substituted ynamide 1a was submitted to these conditions, a 4-amino-5-aryl pyrimidine product (7aa) was obtained in good yield (Fig. 4b). Together with the reactions described previously ( $c f$. Figures 2-3), these results offer an entirely new orthogonal access to either isoquinoline or pyrimidine motifs at will, while unifying this novel, powerful family of formal cycloaddition reactions.
Density functional theory study. We approached the mechanistic study of this reaction performing density functional theory (DFT) calculations of two reaction manifolds: the first leading to isoquinoline products (by modelling the entire pathway introducing a single acetonitrile molecule, see Fig. 5a) and the second leading to pyrimidine adducts (by computing the mechanism with two acetonitrile molecules, see Fig. 5b). The first question that arises is what occurs when all these species are in the presence of the $\mathrm{TfOH}$ promoter, as there are many potential protonation sites. DFT calculations (see Computational details in the Supplementary Figs 65-67) show that protonation would take place preferably on the heteroalkyne partner. Indeed, calculated transition states for the oxazolidinone $(+7.6$ and +6.0$)$ and methylthio $(+5.3$ and +10.7$)$ derivatives (in the presence of either one or two acetonitrile molecules, $\mathbf{I I}_{\mathbf{i}}$ and $\mathbf{I I}_{\mathbf{p}}$, respectively) are much lower than the acetonitrile protonation $\left(+17.0 \mathrm{Kcal} \mathrm{mol}^{-1}\right)$. Furthermore, we confirmed that this protonation takes place regioselectively $\beta$ - to the heteroatom as anticipated, leading to either a keteniminium $\mathbf{I I I}_{\mathbf{i}}$ or ketenethionium III $_{\mathbf{p}}$ species, as the $\mathrm{TfO}^{-}$anion is stabilized by acetonitrile (which in these reactions coincides with the nucleophilic species). In the second mechanistic step, a nucleophilic attack by acetonitrile takes place stereoselectively from the face opposite to the $\beta$-proton due to shielding by $\mathrm{TfO}^{-}$(see $_{\mathbf{I V}} \mathbf{i}$ and $\mathbf{I V}_{\mathbf{p}}$ in Fig. 5). In fact, in both cases the introduction of acetonitrile, giving respectively $\mathbf{V}_{\mathbf{i}}$ and $\mathbf{V}_{\mathbf{p}}$, is more stable than the corresponding $\mathrm{TfO}^{-}$bonded derivative by 6.1 and $7.7 \mathrm{Kcal} \mathrm{mol}^{-1}$ for 


\section{a}

Scope of heteroalkynes in the synthesis of pyrimidines

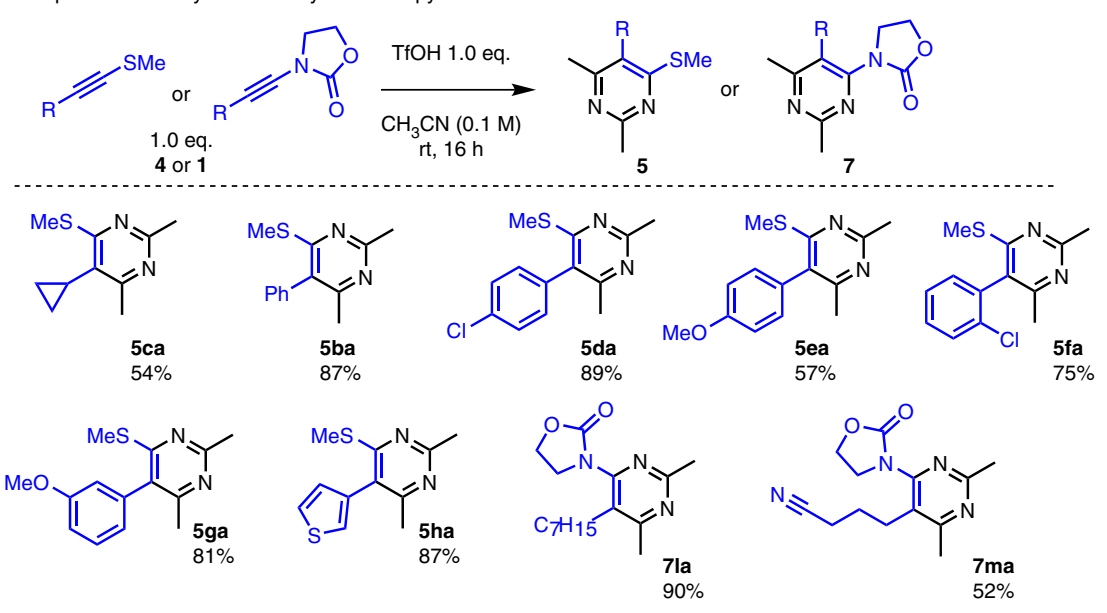

\section{b}

Orthogonality in the synthesis of nitrogen heterocycles

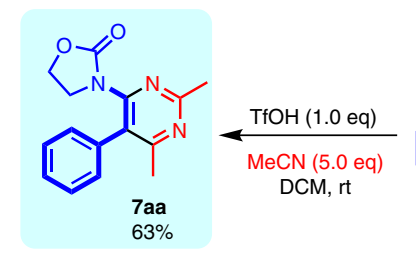

(6.5\% of isoquinoline detected)

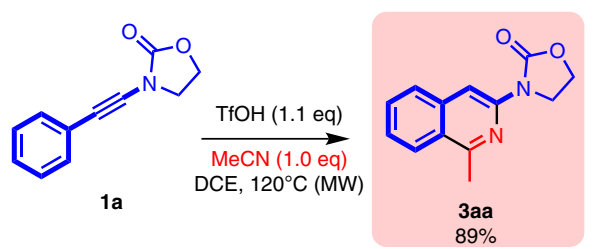

$(<5 \%$ of pyrimidine detected)

Figure 4 | Scope and orthogonality of heterocycles. (a) Scope of heteroalkynes for the synthesis of pyrimidines $\mathbf{5}$ or $\mathbf{7}$. (b) Orthogonality in the synthesis of isoquinolines or pyrimidines from ynamides. See Supplementary Figs 62 and 63 for details.

the oxazolidinone and methylthio derivatives, respectively. Interestingly, and very important for the reaction outcome, in the absence of acetonitrile the $\mathrm{TfO}^{-}$species readily adds to the positively charged intermediate effectively blocking further reaction with acetonitrile. Moreover, we verified that nucleophilic attack by acetonitrile can only take place after the first protonation event as the highest occupied molecular orbital of acetonitrile $(-0.3264 \mathrm{H})$ and the neutral ynamide's lowest unoccupied molecular orbital $(-0.0242 \mathrm{H})$ are energetically too far apart. The protonation process, however, results in an alkyne-centred lowest unoccupied molecular orbital turned by $90^{\circ}$ at -0.2443 $\mathrm{H}$, whereas the highest occupied molecular orbital of the $\mathrm{TfO}^{-}$ counteranion lies at $-0.0742 \mathrm{H}$ (see Supplementary Figs 65 in the Computational details section of the Supplementary Information). A similar trend is observed for the methylthio derivative. These two first processes (protonation + nucleophilic attack of acetonitrile) are common to both pathways. At this juncture, we can separately analyse the mechanisms leading to the isoquinoline and the pyrimidine scaffolds.

Isoquinoline formation. Following the addition of acetonitrile, the $\mathrm{TfO}^{-}$anion immediately adds to the resulting carbocation (as the former lost its prior stabilization by acetonitrile) delivering a neutral and highly stable imino-triflate $\mathbf{V}_{\mathbf{i}}$. The last step consists of a Friedel-Crafts-like cyclization, $\mathbf{V I I}_{\mathbf{i}}$, with further elimination of TfOH giving rise to the isoquinoline skeleton $\left(\mathbf{V I I I}_{\mathbf{i}}\right)$. This final addition process is characterized by high energy-transition states: +30.6 and $+34.5 \mathrm{Kcal} \mathrm{mol}^{-1}$, respectively, for the oxazolidinone and methylthio derivatives $\left(\mathbf{V I}_{\mathbf{i}}\right.$; Fig. 5a). This is why heating is necessary in this case. The higher enthalpic barrier for the methylthio derivative stands in agreement with the experimental findings, as no isoquinoline product is observed for this derivative. In addition, nuclear magnetic resonance studies carried out with the starting heteroalkynes in the presence of $\mathrm{TfOH}$ suggest that the thermal stability of the methylthioderivative is notably low. This could be a determining factor towards the experimental observations.

Pyrimidine formation. In this case, with two acetonitrile molecules (used for simplicity of the model, although the experimentally optimized molar ratio is higher), a different situation arises as a second addition becomes a more probable event. In fact, this process takes place through low energytransition states $\left(+6.5\right.$ and $+7.1 \mathrm{Kcal} \mathrm{mol}^{-1}$, respectively, for the oxazolidinone and methylthio derivatives), $\mathbf{V I}_{\mathbf{p}}$, giving rise to rather stable intermediates $\left(\mathbf{V I I}_{\mathbf{p}}\right.$; see Fig. $\left.5 \mathrm{~b}\right)$. Once the second molecule is added, the system could conceivably undergo a polymerization process with continued further addition of more acetonitrile molecules to the newly generated carbocationic species $\left(+0.546\right.$ and $+0.529 \mathrm{e}^{-}$, respectively, for the oxazolidinone and methylthio derivatives). Instead, the negatively charged $\left(-0.208\right.$ and $-0.210 \mathrm{e}^{-}$, for the oxazolidinone and methylthio derivatives, respectively) $\beta$-carbon atom can attack (VIII $)$ the newly generated carbocation to form an entropically favoured, six-membered pyrimidine ring (after a very exothermic re-aromatization promoted by $\mathrm{TfO}^{-}, \mathbf{I X}_{\mathbf{p}} \rightarrow \mathbf{X}_{\mathbf{p}}$ ). This is the driving force for pyrimidine formation.

A comparative analysis of both calculated mechanisms reveals at a glance that those involving the oxazolidinone derivative proceed generally with lower energies than the thioalkyne one. The main reason for that is the greater stabilization of the positive charge in the former case. In addition, although pathways for 


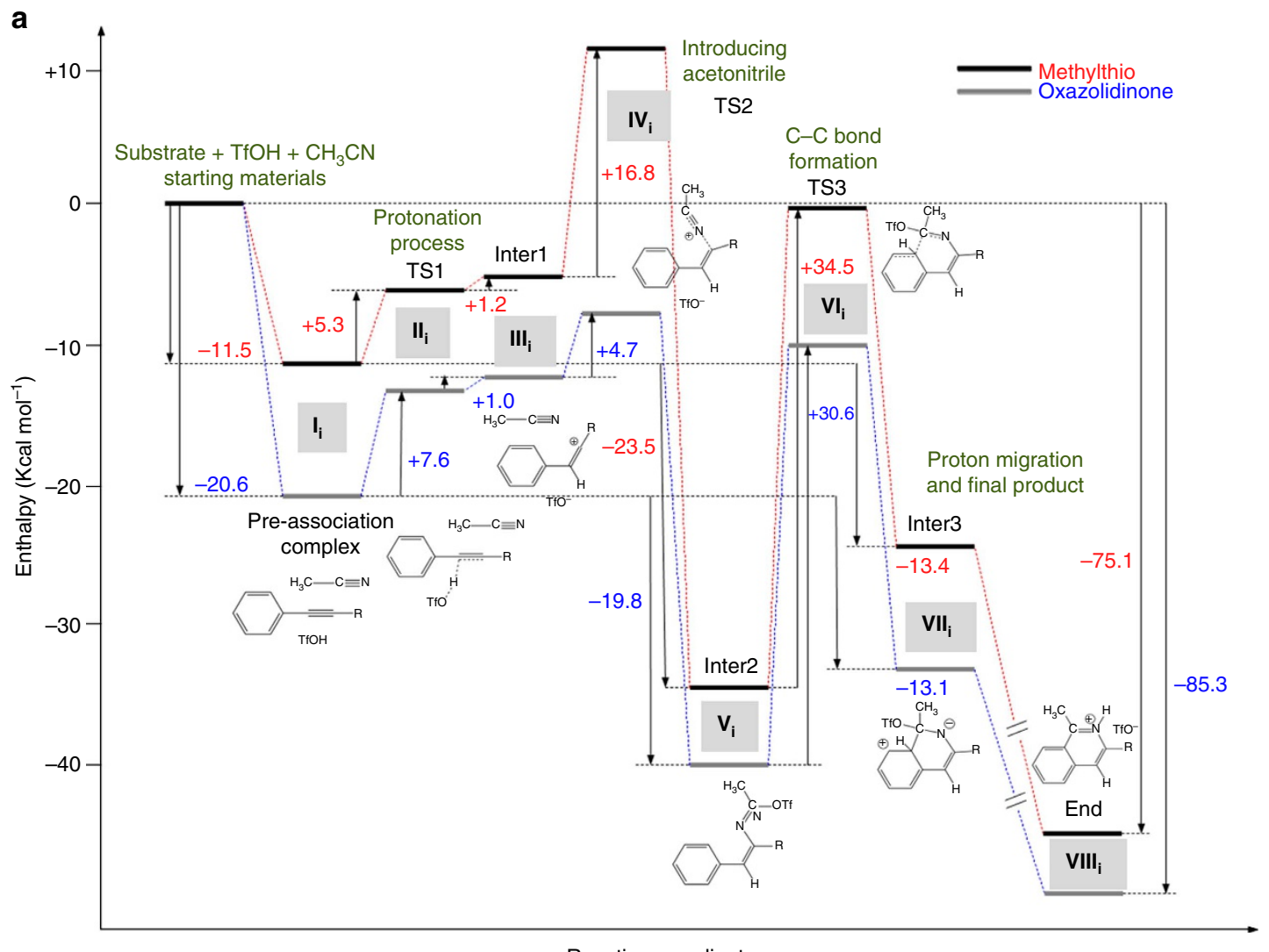

Reaction coordinate

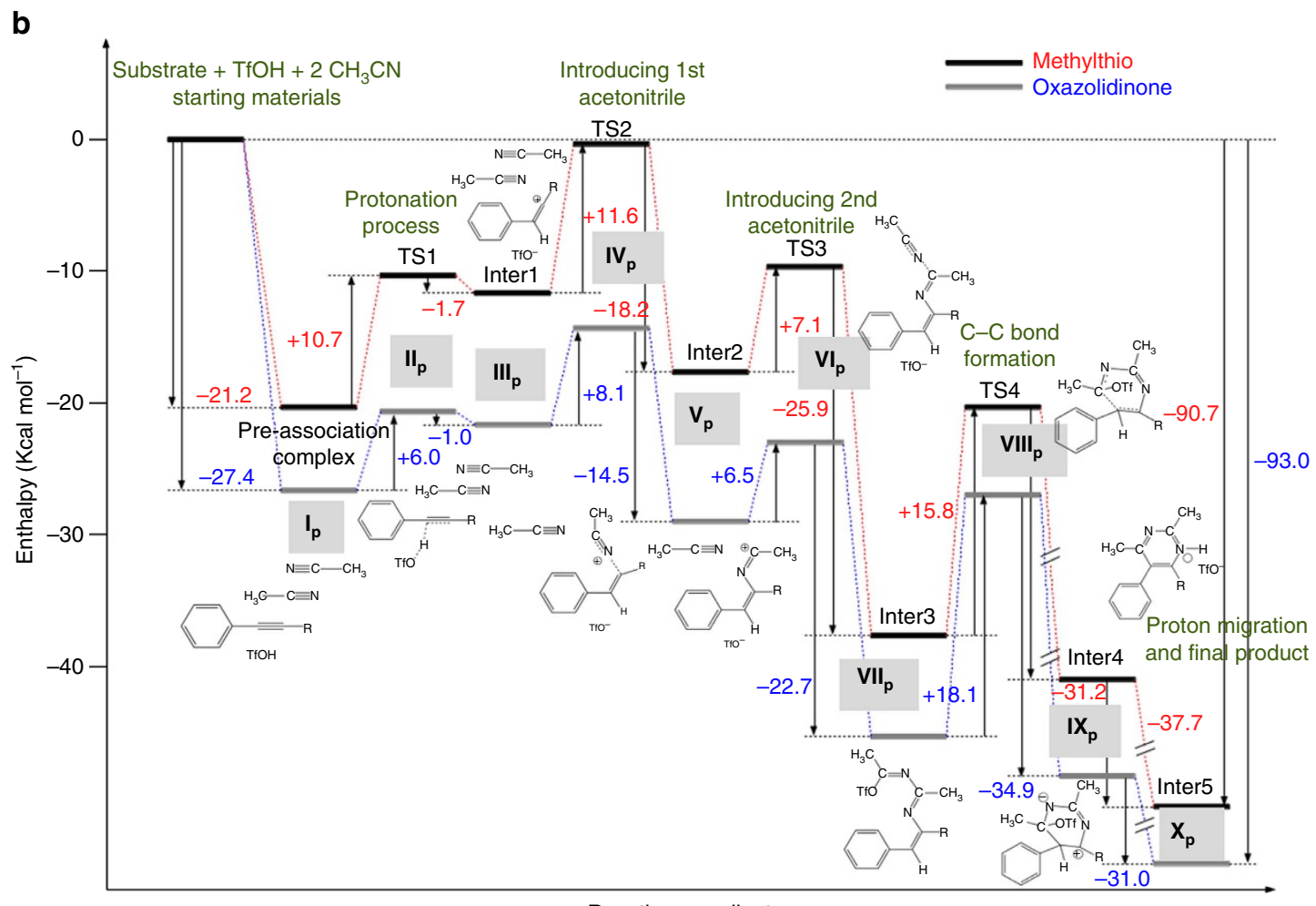

Reaction coordinate

Figure 5 | Energy profiles. Isoquinoline (a, up, i subscript in roman numerals) and pyrimidine (b, down, p subscript in roman numerals) formation for the oxazolidinone (blue) and methylthio (red) derivatives. The corresponding three-dimensional structure sequence is exemplified for the methylthio derivative, for both $\mathbf{a}$ and $\mathbf{b}$ pathways, in Supplementary Figs 66 and 67, respectively 
formation of either heterocycle could exist for both heteroalkynes, the pathway for isoquinoline formation through the thioalkyne derivative (Fig. 5a) has a prohibitive energy barrier when considering the addition of a single acetonitrile molecule. This barrier is much lower in the case of pyrimidine formation (Fig. 5b). This is due to the fact that in the latter case, there is a significant stabilization of the corresponding transition state introduced by the presence of the second acetonitrile molecule. Yet, the transition state for the first acetonitrile attack is $5.2 \mathrm{Kcal} \mathrm{mol}^{-1}$ higher in the case of isoquinoline formation. In this value, $3.2 \mathrm{Kcal} \mathrm{mol}^{-1}$ are purely due to the stabilization offered by the second acetonitrile molecule, as calculations made considering cationic structures (namely just the substrate and the
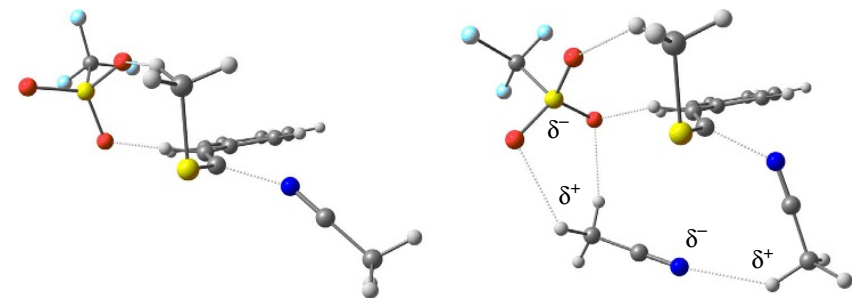

Figure 6 | Transition states for the first acetonitrile addition. Isoquinoline (left) and pyrimidine (right) pathways, the latter presenting additional cyclic, electrostatic stabilizing interactions. acetonitrile molecule(s) without the $\mathrm{TfO}^{-}$species) show this same energy difference. Therefore, the remaining $2 \mathrm{Kcal} \mathrm{mol}^{-1}$ should derive from stabilization by the counteranion (which is present in our mechanistic studies) through cooperative cyclic $\delta^{+} \cdot \delta^{-}$interactions between the different molecular units (see Fig. 6). In fact, we expect this transition state to be very low-lying, taking into account more solvent molecules. The preceding mechanistic analysis also permits a rationalization of why isoquinoline synthesis (formal [4+2]; (refs 49-52)) requires high temperatures (highest energy barriers), whereas pyrimidine formation (formal $[2+2+2]$ ) typically occurs at room temperature.

In both cases, stoichiometry plays a crucial role and imposes the final result, as both pathways are irreversible. In the presence of several molecules of acetonitrile (Fig. 5b), the corresponding transition state for a real $[2+2+2]$ approximation is either transition state $\mathbf{I V}_{\mathbf{p}}$ or $\mathbf{V I}_{\mathbf{p}}$, depending on the initial geometry conditions. These transition state also appear in a sequential pathway (as described in Fig. 5), thus indicating a natural direction for this molecular set. In the case of isoquinoline formation (Fig. 5a), the reaction of the unique acetonitrile molecule (imposed by stoichiometry) creates a positive charge that is readily neutralized by the negatively charged triflate present in the surrounding (as a remnant from the initial protonation event). The latter reaction should be faster than the time required for another acetonitrile molecule to approach, to<smiles>COc1ccc(Cc2nc(N3CCOC3=O)cc3cc(OC)c(OC)cc23)cc1OC</smiles>

3di<smiles>COc1ccc(CC2NCCc3cc(OC)c(OC)cc32)cc1OC</smiles>

( \pm -Norlaudanosine 8

b

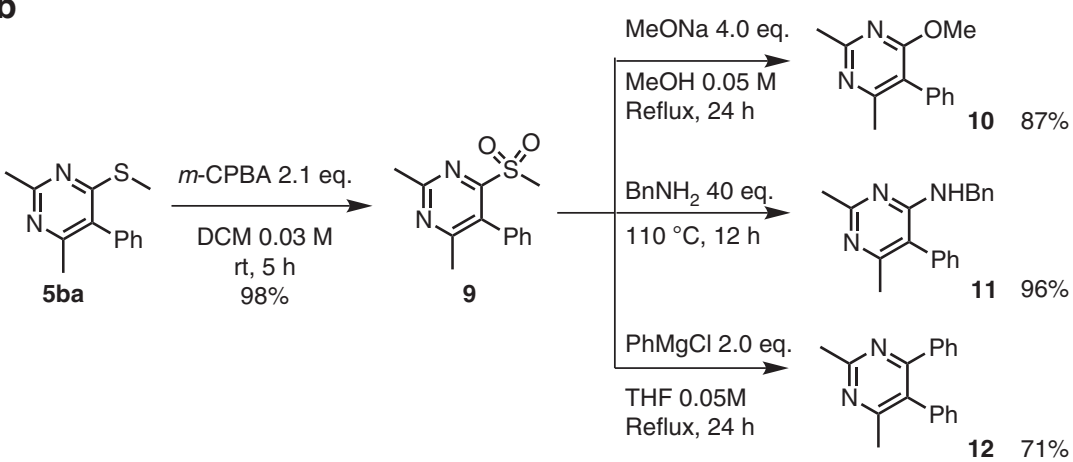

C

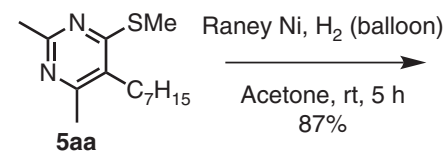<smiles>CCc1cnc(C)nc1C</smiles>

d

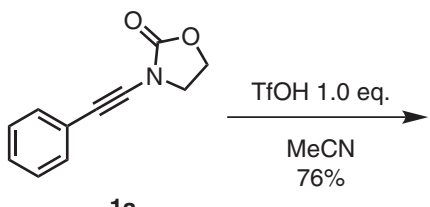

(1 g.)

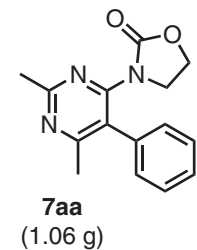

Figure 7 | Synthetic application and modification. (a) Preparation of norlaudanosine $\mathbf{8}$ by hydrogenation of 3di. (b) Transformations of compound $\mathbf{5 b a}$ (c) Reductive desulfurization of compound $\mathbf{5 a a}$. (d) Pyrimidine $\mathbf{7}$ aa can be prepared in gram scale. 
follow pathway B. Once triflate blocks this position, a quite stable intermediate $\left(\mathbf{V}_{\mathbf{i}}\right)$ is formed and no additional acetonitrile molecules can be added.

It is noteworthy that although all the computed reaction pathways would only require a catalytic amount of $\mathrm{TfOH}$ to proceed (owing to its regeneration on aromatization, vide supra), the most stable final product in either pathway is the corresponding nitrogen-protonated heterocycle (readily converted into the experimentally isolated products following basic workup). This neatly accommodates the experimental need for stoichiometric amounts of acid, to obtain high yields.

Further studies. Given the prevalence of isoquinoline motifs in the core of bioactive molecules ${ }^{53-55}$, we were eager to showcase the synthetic utility of our products (Fig. 7). The tetramethoxy adduct 3di, carrying two electronically differentiated fused rings in its isoquinoline system, could be hydrogenated in acetic acid to deliver ( \pm )-norlaudanosine 8 (Fig. 7a). Removal of the oxazolidinone takes place under these conditions analogously to previous work by Glorius et al. ${ }^{56}$ on related pyridines and quinolones ${ }^{57}$. It is noteworthy that the use of a chiral 2-oxazolidone analogue, as in $\mathbf{3 h i}$, enabled the hydrogenation to proceed with some level of asymmetric induction ( $55 \%$ e.e.). See Supplementary Fig. 60 for more detail.

The (methylthio)pyrimidine 5 ba could be easily oxidized to the corresponding methylsulfonyl derivative $\mathbf{9}$, in which the methylsulfonyl group is available for substitution. As shown in Fig. 7b, this can be achieved by the action of alcohols, amines or Grignard reagents, delivering substituted pyrimidines 10-12 in very good to excellent yields.

In addition, Raney-Ni-mediated hydrogenation of $\mathbf{5 a a}$ smoothly excises the sulfide residue to afford the 2,5,6trisubstituted pyrimidine 13 in $87 \%$ yield (Fig. 7c). These simple transformations outline the versatility and usefulness of the methods reported herein. Moreover, the reaction can be readily carried out in gram scale (Fig. $7 \mathrm{~d}$ ).

\section{Discussion}

A family of reactions selectively leading to isoquinoline and pyrimidine motifs has been developed, by Brønsted acidpromoted regioselective merger of alkynes and nitriles. These methodologies benefit from the strategic use of readily available nitriles as the $\mathrm{C}-\mathrm{N}$ sources. Most importantly, the orthogonality of the methods enables the preparation of either family of heterocycles from the same starting materials. The practicality of these metal-free formal cycloadditions is illustrated by the large scope of alkynes and nitriles that can be employed. DFT calculations reveal the crucial role of $\mathrm{TfOH}$ and the reaction stoichiometry in these processes. With one equivalent of acetonitrile, the preferred pathway leads to isoquinoline products through a Friedel-Crafts-like process; with larger amounts of nitrile, a second addition is allowed en route to the formation of a pyrimidine derivative. Furthermore, subtle differences between the classes of heteroalkynes employed control which products can be formed. We believe that the simple yet powerful heterocycle syntheses presented here will be eagerly adopted into the repertoire of synthetic chemistry.

\section{Methods}

Full experimental details, characterization of compounds, Cartesian coordinates and energies of all the structures appearing in Supplementary Figs 66 and 67, and computational details can be found in the Supplementary Information (Supplementary Figs 1-67 and Supplementary Methods).

\section{References}

1. Joule, J. A. \& Mills, K. Heterocyclic Chemistry 5th edn 194-200 (John Wiley \& Sons, Ltd, 2010).

2. Iranshahy, M., Quinn, R. J. \& Iranshahi, M. Biologically active isoquinoline alkaloids with drug-like properties from the genus Corydalis. RSC Adv. 4, 15900-15913 (2014).

3. Bentley, K. W. $\beta$-Phenylethylamines and the isoquinoline alkaloids. Nat. Prod. Rep. 23, 444-463 (2006).

4. Su, Y. J. et al. Highly efficient red electrophosphorescent devices based on iridium isoquinoline complexes: remarkable external quantum efficiency over a wide range of current. Adv. Mater. 15, 884-888 (2003).

5. Ho, C.-L. et al. Red-light-emitting iridium complexes with hole-transportingn 9-arylcarbazole moieties for electrophosphorescence efficiency/color purity trade-off optimization. Adv. Funct. Mater. 18, 319-331 (2008).

6. Walker, S. R., Carter, E. J., Huff, B. C. \& Morris, J. C. Variolins and related alkaloids. Chem. Rev. 109, 3080-3098 (2009).

7. Lagoja, I. M. Pyrimidine as constituent of natural biologically active compounds. Chem. Biodivers. 2, 1-50 (2005).

8. Köytepe, S., Paşahan, A., Ekinci, E. \& Seçkin, T. Synthesis, characterization and $\mathrm{H}_{2} \mathrm{O}_{2}$-sensing properties of pyrimidine-based hyperbranched polyimides. Eur. Polym. J. 41, 121-127 (2005).

9. Gompper, R., Mair, H.-J. \& Polborn, K. Synthesis of oligo(diazaphenyls). Tailor-made fluorescent heteroaromatics and pathways to nanostructures. Synthesis (Mass) 696-718 (1997).

10. He, R., Huang, Z.-T., Zheng, Q.-Y. \& Wang, C. Isoquinoline skeleton synthesis via chelation-assisted C-H activation. Tetrahedron Lett. 55, 5705-5713 (2014).

11. Shi, Z., Koester, D. C., Boultadakis-Arapinis, M. \& Glorius, F. Rh(III)-catalyzed synthesis of multisubstituted isoquinoline and pyridine $\mathrm{N}$-oxides from oximes and diazo compounds. J. Am. Chem. Soc. 135, 12204-12207 (2013).

12. Zhao, D., Lied, F. \& Glorius, F. Rh(III)-catalyzed C-H functionalization/ aromatization cascade with 1,3-dienes: a redoxneutral and regioselective access to isoquinolines. Chem. Sci. 5, 2869-2873 (2014).

13. Roesch, K. R., Zhang, H. \& Larock, R. C. Synthesis of isoquinolines and pyridines by the palladium-catalyzed iminoannulation of internal alkynes. J. Org. Chem. 66, 8042-8051 (2001).

14. Fischer, D. et al. Iodine-mediated electrophilic cyclization of 2-alkynyl-1methylene azide aromatics leading to highly substituted isoquinolines and its application to the synthesis of Norchelerythrine. J. Am. Chem. Soc. 130, 15720-15725 (2008) and references therein.

15. Gilmore, C. D., Allan, K. M. \& Stoltz, B. M. Orthogonal synthesis of indolines and isoquinolines via aryne annulation. J. Am. Chem. Soc. 130, 1558-1559 (2008).

16. Castillo, J.-C., Quiroga, J., Abonia, R., Rodriguez, J. \& Coquerel, Y. The aryne aza-Diels-Alder reaction: flexible syntheses of isoquinolines. Org. Lett. 17, 3374-3377 (2015).

17. Coppola, A., Sucunza, D., Burgos, C. \& Vaquero, J. J. Isoquinoline synthesis by heterocyclization of tosylmethyl isocyanide derivatives: total synthesis of mansouramycin B. Org. Lett. 17, 78-81 (2015).

18. Mrtínez, A. G., Fernández, A. H., Vilchez, D. M., Gutiérrez, M. L. L. \& Subramanian, L. R. A new easy one-step synthesis of isoquinoline derivatives from substituted phenylacetic esters. Synlett. 1993, 229-230 (1993).

19. Hill, M. D. \& Movassaghi, M. New strategies for the synthesis of pyrimidine derivatives. Chem. Eur. J. 14, 6836-6844 (2008) and references therein.

20. Movassaghi, M. \& Hill, M. D. Single-step synthesis of pyrimidine derivatives. J. Am. Chem. Soc. 128, 14254-14255 (2006).

21. Martínez, A. G. et al. On the mechanism of the reaction between ketones and trifluoromethanesulfonic anhydride. An improved and convenient method for the preparation of pyrimidines and condensed pyrimidines. J. Org. Chem. 57, 1627-1630 (1992) and references therein.

22. Herrera, A., Martínez-Álvarez, R., Chioua, M., Chioua, R. \& Sánchez, Á. On the regioselectivity in the reaction of aliphatic ketones and aromatic nitriles. Regiospecific synthesis of alkylarylpyrimidines. Tetrahedron 58, 10053-10058 (2002).

23. Martínez, A. G. et al. Sterically hindered bases. Synthesis of 2,4,6-trisubstituted pyrimidine. Synthesis (Mass) 881-882 (1990) and references therein.

24. Karad, S. N. \& Liu, R.-S. Regiocontrolled gold-catalyzed $[2+2+2]$ cycloadditions of ynamides with two discrete nitriles to construct 4-aminopyrimidine cores. Angew. Chem. Int. Ed. 53, 9072-9076 (2014).

25. Evano, G., Coste, A. \& Jouvin, K. Ynamides: versatile tools in organic synthesis. Angew. Chem. Int. Ed. 49, 2840-2859 (2010).

26. DeKorver, K. A. et al. Ynamides: a modern functional group for the new millennium. Chem. Rev. 110, 5064-5106 (2010).

27. Wang, X.-N. et al. Ynamides in ring forming transformations. Acc. Chem. Res. 47, 560-578 (2014).

28. Li, L. et al. Zinc-catalyzed alkyne oxidation/C-H functionalization: highly site selective synthesis of versatile isoquinolones and $\beta$-carbolines. Angew. Chem. Int. Ed. 54, 8245-8249 (2015). 
29. Shu, C. et al. Generation of $\alpha$-imino gold carbenes through gold-catalyzed intermolecular reaction of azides with ynamides. J. Am. Chem. Soc. 137, 9567-9570 (2015).

30. Theunissen, C. et al. Keteniminium ion-initiated cascade cationic polycyclization. J. Am. Chem. Soc. 136, 12528-12531 (2014).

31. Peng, B., Huang, X., Xie, L.-G. \& Maulide, N. A Brønsted acid-catalyzed redox arylation. Angew. Chem. Int. Ed. 53, 8718-8721 (2014).

32. Xin, Z., Kramer, S., Overgaard, J. \& Skrydstrup, T. Access to 1,2-dihydroisoquinolines through gold-catalyzed formal $[4+2]$ cycloaddition. Chem. Eur. J. 20, 7926-7930 (2014).

33. Minko, Y., Pasco, M., Lercher, L., Botoshansky, M. \& Marek, I. Forming all-carbon quaternary stereogenic centres in acyclic systems from alkynes. Nature 490, 522-526 (2012).

34. Ding, S. et al. Highly regio- and stereoselective hydrosilylation of internal thioalkynes under mild monditions. Angew. Chem. Int. Ed. 54, 5632-5635 (2015).

35. Ding, S., Jia, G. \& Sun, J. Iridium-catalyzed intermolecular azide-alkyne cycloaddition of internal thioalkynes under mild conditions. Angew. Chem. Int. Ed. 53, 1877-1880 (2014).

36. Huang, K.-H. \& Isobe, M. Highly regioselective hydrosilylation of unsymmetric alkynes using a phenylthio directing group. Eur. J. Org. Chem. 4733-4740 (2014) and references therein.

37. Frei, R. et al. Fast and highly chemoselective alkynylation of thiols with hypervalent iodine reagents enabled through a low energy barrier concerted mechanism. J. Am. Chem. Soc. 136, 16563-16573 (2014).

38. Favre, A. \& Fourrey, J.-L. Structural probing of small endonucleolytic ribozymes in solution using thio-substituted nucleobases as intrinsic photolabels. Acc. Chem. Res. 28, 375-382 (1995).

39. Dubbaka, S. R. \& Vogel, P. Organosulfur compounds: electrophilic reagents in transition-metal-catalyzed carbon-carbon bond-forming reactions. Angew. Chem. Int. Ed. 44, 7674-7684 (2005).

40. Prokopcová, H. \& Kappe, C. O. The Liebeskind-Srogl C-C cross-coupling reaction. Angew. Chem. Int. Ed. 48, 2276-2286 (2009)

41. Pan, F. \& Shi, Z.-J. Recent advances in transition-metal-catalyzed C-S activation: from thioester to (hetero)aryl thioether. ACS Catal. 4, 280-288 (2014).

42. Melzig, L., Metzger, A. \& Knochel, P. Pd- and Ni-catalyzed cross-coupling reactions of functionalized organozinc reagents with unsaturated thioethers. Chem. Eur. J. 17, 2948-2956 (2011) and references therein.

43. Chopade, P. R. \& Louie, J. [2+2+2] Cycloaddition reactions catalyzed by transition metal compleyes. Adv. Synth. Catal. 348, 2307-2327 (2006).

44. Suzuki, H. \& Abe, H. A simple cyclization route to some 4-substituted 3-aminoisoquinolines. Synthesis (Mass) 763-765 (1995) and references therein.

45. Neumeyer, J. L., Weinhardt, K. K., Carrano, R. A. \& McCurdy, D. H. Isoquinolines 3. 3-aminoisoquinoline derivatives with central nervous system depressant activity. J. Med. Chem. 16, 808-813 (1973).

46. Straub, A. et al. NO-independent stimulators of soluble guanylate cyclase. Bioorg. Med. Chem. Lett. 11, 781-784 (2001).

47. Axon, J., Chakravarty, S., Dugar, S., Mcenroe, G. \& Murphy, A. Inhibitors of TFGbeta. PCT Int. Appl. WO 2004/024159 A1 (2004).

48. Mciver, E. G. et al. Pyrimidine derivatives capable of inhibiting one or more kinases. APCT Int. ppl. WO 2009/122180 Al (2009).

49. Barluenga, J., Fernández-Rodríguez, M. Á., García-García, P. \& Aguilar, E. Gold-catalyzed intermolecular hetero-dehydro-diels - alder cycloaddition of captodative dienynes with nitriles: a new reaction and regioselective direct access to pyridines. J. Am. Chem. Soc. 130, 2764-2765 (2008).

50. Wessig, P. \& Müller, G. The dehydro-diels - alder reaction. Chem. Rev. 108, 2051-2063 (2008).

51. Fernández-García, J. M., Fernández-Rodríguez, M. Á. \& Aguilar, E. Catalytic intermolecular hetero-dehydro-Diels-Alder cycloadditions: regio- and diasteroselective synthesis of 5,6-dihydropyridin-2-ones. Org. Lett. 13, 5172-5175 (2011).

52. Hoye, T. R., Baire, B., Niu, D., Willoughby, P. H. \& Woods, B. P. The hexadehydro-Diels-Alder reaction. Nature 490, 208-212 (2012).

53. Chrzanowska, M. \& Rozwadowska, M. D. Asymmetric synthesis of isoquinoline alkaloids. Chem. Rev. 104, 3341-3370 (2004).

54. Scott, J. D. \& Williams, R. M. Chemistry and biology of the tetrahydroisoquinoline antitumor antibiotics. Chem. Rev. 102, 1669-1730 (2002).

55. Kartsev, V. G. Natural compounds in drug discovery. Biological activity and new trends in the chemistry of isoquinoline alkaloids. Med. Chem. Res. 13, 325-336 (2004).

56. Glorius, F., Spielkamp, N., Holle, S., Goddard, R. \& Lehmann, C. W. Efficient asymmetric hydrogenation of pyridines. Angew. Chem. Int. Ed. 43, 2850-2852 (2004).

57. Heitbaum, M., Frchlich, R. \& Glorius, F. Diastereoselective hydrogenation of substituted quinolines to enantiomerically pure decahydroquinolines. $A d v$ Synth. Catal. 352, 357-362 (2010).

\section{Acknowledgements}

We are grateful to the European Research Council (ERC StG 278872 to N.M.) and the University of Vienna for support of this work. We acknowledge Dr Michael J. Fink and Professor Marko D. Mihovilovic (Vienna University of Technology) for hydrogenation reactions and helpful discussions. The computational results have been achieved in part using the Vienna Scientific Cluster (VSC).

\section{Author contributions}

L.-G.X. and N.M. planned the project. L.-G.X. and S.N. carried out the experiments and analysed the data. A.J.M. and L.G. carried out the DFT analysis. L.-G.X., A.J.M., S.N., L.G. and N.M. wrote the manuscript.

\section{Additional information}

Accession codes: The X-ray crystallographic coordinates for structures reported in this study have been deposited at the Cambridge Crystallographic Data Centre (CCDC), under deposition numbers CCDC 1423496. These data can be obtained free of charge from The Cambridge Crystallographic Data Centre via www.ccdc.cam.ac.uk/data_request/cif.

Supplementary Information accompanies this paper at http://www.nature.com/ naturecommunications

Competing financial interests: The authors declare no competing financial interests

Reprints and permission information is available online at http://npg.nature.com/ reprintsandpermissions/

How to cite this article: Xie, L.-G. et al. Metal-free intermolecular formal cycloadditions enable an orthogonal access to nitrogen heterocycles. Nat. Commun. 7:10914 doi: $10.1038 /$ ncomms10914 (2016).

(c) (i)

This work is licensed under a Creative Commons Attribution 4.0 International License. The images or other third party material in this article are included in the article's Creative Commons license, unless indicated otherwise in the credit line; if the material is not included under the Creative Commons license users will need to obtain permission from the license holder to reproduce the material To view a copy of this license, visit http://creativecommons.org/licenses/by/4.0/ 